the East Aegean Islands is continuing to develop. Publication of this series of volumes from the University of Edinburgh Press began in 1965 and still proceeds. Similarly, to the south of the Caspian, K. H. Rechinger, based in Vienna, continues to work upon Flora Iranica, which commenced publication in the early 1960s.

A further, well advanced project is Flora Palaestina which is being published by the Israel Academy of Sciences and Humanities, Jerusalem. The recently published third part of this series covers the Ericaceae to Compositae, and the geographical range of the Flora encompasses Israel and the west and east banks of the Jordan rift valley, south to Elat and Aqaba. There are keys to genera and species of Palestinian plants plus detailed descriptions of each species. These descriptions include notes concerning the ecology and habitats of the plants, as well as their geographical range within the study area and their full world range. These are useful notes which are not generally found in Flora Europaea because of pressure on space.

Each part of Flora Palaestina is accompanied by a volume of plant drawings which are of very high artistic quality and botanical accuracy. Five artists have been employed in this task, but a commendable uniformity of style has been maintained. Since a fair proportion of the plants illustrated are widely distributed in the eastern Mediterranean, these drawings will prove useful and interesting to European botanists as well as to the Israelis. Which leads one to the question whether a similar set of illustrations could be prepared to accompany Flora Europaea? Herculean, perhaps, but nevertheless a profitable exercise.

Peter D. Moore is Senior Lecturer in Plant Sciences at King's College, University of London, UK.

\section{Pore structure and flow modelling}

\section{J. Gunn}

Porous Media. By F. A. L. Dullien. Pp. 396. (Academic: New York and London, 1980.) $\$ 42, £ 23.60$.

IN THE preface Professor Dullien gives the primary purpose of this book as "presenting in an organised manner the most pertinent information available on the role of pore structure and then putting it to use in the interpretation of experimental data and the results of model calculations". In pursuit of these aims the book is divided into two principal parts, the first of which is devoted to the description of pore structure, the second to flow properties of porous media.

Work on pore structure is described in two 70-page chapters. In Chapter 2, "On Capillarity in Porous Media', the description of capillary pressure measurements in porous media is followed by a description of pore network models of capillary tubes and capillary pressure functions generated by network models. In Chapter 3 porosity and permeability are defined, the measurement of pore size and pore size distribution is considered, and the measurements of pore size distribution are described for a range of materials. Singlephase flow in porous media is considered in Chapter 4 and includes a survey of some of the available correlations between flow and pressure gradient, statistical permeability models and network models, with sections on gas flow and diffusion, and nonNewtonian flow. The description of multiphase flow of immiscible fluids, Chapter 6, is mainly directed to the flow of oil and water through both consolidated porous solids and unconsolidated solids, while the last major part of the book is Chapter 7 on miscible displacement and dispersion.

The emphasis of the book is on fundamental studies of interest in petroleum recovery and production, although topics such as the formation, flow and dispersion of micro-emulsions that are of interest in secondary recovery are not considered in detail. The book would have been more helpful if the description of models for flow and dispersion in porous media was more critical. The most important criteria for the success of a model description are that the model should be well founded, and that it should describe experimental phenomena without invoking significant further measurements to define the parameters of the model when applied to a particular situation. Some of the model descriptions of dispersion in porous media, although considered in some detail in the book, have not been shown to relate to experimental measurements of dispersion, while the use of a bivariate pore size distribution to estimate permeability requires more experimental work than a direct measurement!

However, for those who have already been introduced to the study, the publication of a research monograph on flow in porous media is timely because of the economic importance of techniques for secondary oil recovery. Further, it is very useful to have this comprehensive survey available as an aid in process development and in the mathematical modelling of field flows.

D. J. Gunn is Reader in Chemical Engineering at the Univeristy College of Swansea, UK.
Macromolecules in 3D

\section{Colin Blake}

Teaching Aids for Macromolecular Structure. Compiled by Richard J. Feldmann and David H. Bing. Instructor manual pp.114 with 116 stereoslides; student kit 49 stereoslides with viewers. (Taylor-Merchant: New York, 1980.) Instructor kit $\$ 210$, student kit $\$ 15$.

THE APPLICATION of the X-ray diffraction technique to crystalline and paracrystalline biological materials has produced a solid body of information on the structure and function of many biological macromolecules. After an initial period of scepticism concerning the significance of the results, this material has now become reasonably well integrated into the broad field of biochemistry, and is now routinely taught at the undergraduate level, not only to biochemists, but also to those in other more biological or physical sciences. Teaching this material is however fraught with problems, most of which derive from the sheer complexity of biological macromolecules. Although the repetitive structures of DNA, the $\alpha$-helix, $\beta$-sheet and even the collagen helix are fairly easy to visualize, the molecules of t-RNA, supercoiled DNA and most proteins and enzymes are much more difficult to illustrate. To overcome these difficulties a variety of short cuts are no doubt taken; the most extreme is to ignore all of an enzyme except its catalytic site, which at best does less than justice to enzymes and at worst gives a completely distorted view of the raison d'être of the subject, the attempt to understand the characteristic integration of structure and function exhibited by biological macromolecules. To say this does not necessarily imply criticism of the people who use the short cuts; it probably has much more to do with the sheer difficulty of producing teaching material that shows the extent of the structure function integration. Even with a complete set of plastic and wire models, and mono and stereo slides there are problems: models are either too small or too complex to point out particular features to a class of students, and slides, even those in stereo, have of necessity to be simplified and cannot show all the features of a complete molecule. And, of course, many departments are not comprehensively equipped in this way.

With these problems as an overt background, a new aid for teaching macromolecular structure has just become available. Called TAMS, an acronym for Teaching Aids for Macromolecular Structure, it has been compiled by Richard Feldmann of NIH and David Bing of The Harvard Medical School. TAMS consists of a large ring-back folder containing a 